\title{
Challenges and Opportunities for the Next Generation of Photon Regeneration Experiments
}

\author{
Andreas Ringwald \\ Deutsches Elektronen-Synchrotron DESY, Notkestrasse 85, 22607 Hamburg, Germany
}

\begin{abstract}
Photon regeneration experiments searching for signatures of oscillations of photons into hypothetical very weakly interacting ultra-light particles, such as axions, axion-like and hiddensector particles, have improved their sensitivity considerably in recent years. Important progress in laser and detector technology as well as recycling of available magnets from accelerators may allow a big further step in sensitivity such that, for the first time, laser light shining through a wall experiments will explore territory in parameter space that has not been excluded yet by astrophysics and cosmology. We review these challenges and opportunities for the next generation experiments.
\end{abstract}

Keywords: Low energy experimental particle physics, extensions of the Standard Model, axions, extra gauge bosons, hidden-sector matter particles

PACS: $14.80 .-\mathrm{j}, 14.70 . \mathrm{Pw}, 12.60 . \mathrm{Cn}, 12.20 . \mathrm{Fv}$

Over the last few years, it became more and more clear that precision experiments exploiting low-energy photons may yield information on particle physics complementary to experiments at high-energy colliders, in particular on the possible existence of new very weakly interacting sub-eV particles (WISPs), such as axions [1], axion-like particles (ALPs), and hidden-sector particles (hidden photons [2], minicharged particles [3]), predicted in many extensions of the Standard Model [4, 5]. The report by the laser polarization experiment PVLAS of the observation of an anomalously large rotation of the polarization plane of photons after the passage through a magnetic field [6] - which may be interpreted as evidence for photon disappearance due to conversion into WISPs $[7,8,9,10,11]$ - provided the impetus for a number of laser light shining through a wall (LSW) experiments. The latter are searching for photon $\rightarrow$ WISP $\rightarrow$ photon conversions (cf. Fig. 1) rather than solely for disappearence, to perform an
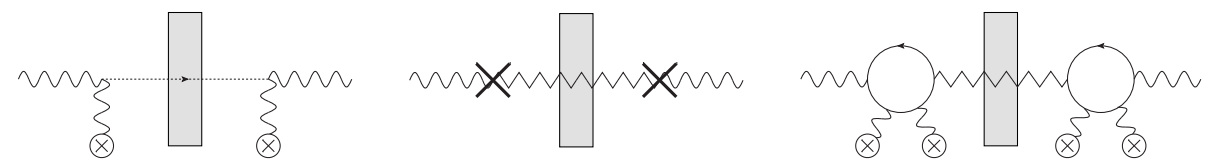

FIGURE 1. In LSW experiments, laser photons are sent along a beam onto a wall where they are absorbed. Some of the photons may converted into WISPs that propagate freely through the wall and reconvert into photons after the wall. LSW may occur due to various processes beyond the Standard Model: $\gamma \leftrightarrow$ ALP oscillations in a background magnetic field [12, 13, 14] (left), $\gamma \leftrightarrow \gamma^{\prime}$ oscillations facilitated by a non-zero mass of the hidden photon $\left(\gamma^{\prime}\right)$ [2] (middle), and $\gamma \leftrightarrow \gamma^{\prime}$ oscillations facilitated by virtual mini-charged particles in a background magnetic field [10, 11] (right). (From Ref. [4].)

independent test of the WISP hypothesis $[15,16,17,18,19,20,21]$ und to improve the constraints from the pioneering experiment BFRT [22] by about an order of magnitude in the WISP-photon coupling (cf. Fig. 2). Moreover, the momentum gained by these experiments towards the establishment of a new low-energy frontier of particle physics 

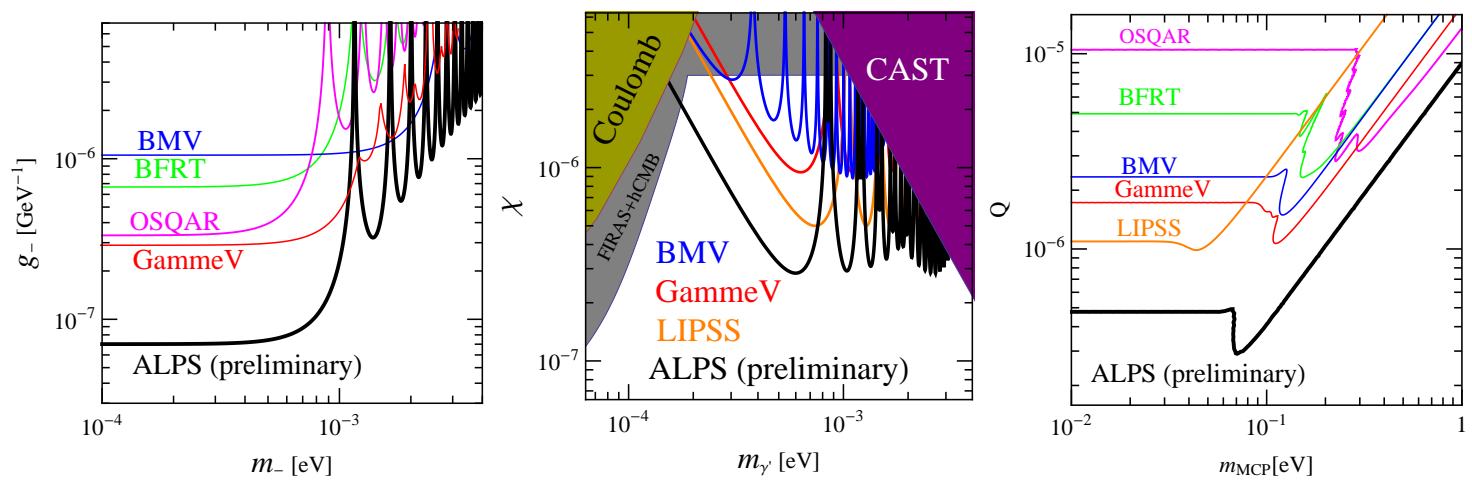

FIGURE 2. Upper bounds from LSW experiments for couplings of pseudoscalar axion-like particles (two photon coupling $g$; left panel), massive hidden photons (kinetic mixing $\chi$; middle panel), and massless hidden photons with an additional minicharged particle (charge $Q=\chi$; right panel). The results from ALPS are preliminary. Compilation from Ref. [23].

turned out to be conserved even though the original motivation disappeared: the PVLAS collaboration could not reestablish their first observation after an upgrade of their apparatus [24]. This is in-line with the finding of the above mentioned LSW experiments.

Now, the planning for the next generation of photon regeneration experiments has started. At this stage, it seems to be very helpful to identify targets in WISP parameter space upon which the next generation of experiments can shoot. In this context, one can clearly identify both

- challenges: increase sensitivity beyond astro, cosmo, and other lab bounds, and

- opportunities: test WISP interpretation of hints for cosmic photon regeneration,

that we will discuss in detail in the following.

For hidden photons, laser LSW experiments are in a comfortable position, as is illustrated in Figs. 2 (middle) and 3: already by now, they are exploring previously untouched parameter space, bearing therefore the greatest immediate discovery potential. The cosmo constraint arising from the upper limit on the effective number of relativistic degrees of freedom contributing to the cosmic radiation density in the era between big bang nucleosynthesis and recombination [26] (grey area in Fig. 2 (middle)) as well as the constraint arising from a search for photon regeneration due to solar hidden photons in the CAST helioscope [27] (purple area in Fig. 2 (middle)) are not competitive with LSW limits in the $\sim$ meV mass range.

This is, however, only true if there is no light physical hidden Higgs particle involved, i.e. if the hidden photon gets its mass from a Stückelberg mechanism. Otherwise, if the hidden photon mass arises via a Higgs mechanism, the physical hidden Higgs effectively acts as a minicharged particle, with charge $Q=\chi e_{h} / e$, where $e_{h}$ is the gauge coupling of the hidden photon, and the strong astro bound $Q \lesssim 10^{-14}$, for a sub-keV hidden Higgs mass, inferred from the lifetime of red giants applies [28]. In particularly well motivated LARGE volume string compactifications, the gauge coupling of the hidden photon can be hyperweak, i.e. diluted due to the volume of the extra dimensions, to $e_{h} \sim$ $10^{-6}$, for a volume corresponding to an intermediate string scale $M_{s} \sim 10^{9} \mathrm{GeV}$ [29]. 


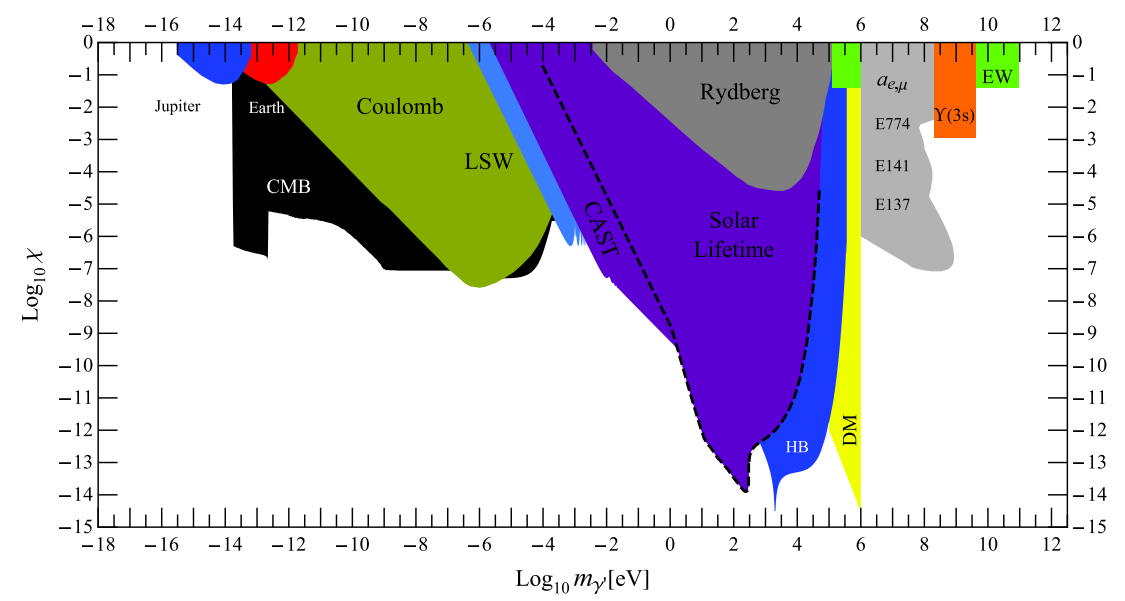

FIGURE 3. Summary of astrophysical, cosmological and laboratory constraints for hidden photons (kinetic mixing $\chi$ vs. mass $m_{\gamma^{\prime}}$ ) (Adapted from Ref. [25], where also details can be found.).

Therefore, the limit on minicharged particles excludes $\chi \gtrsim$ few $\times 10^{-9}$, at low masses, $m_{\gamma^{\prime}} \sim m_{H_{h}} \lesssim \mathrm{keV}$ (cf. Fig. 4). Thus, the discovery potential for hidden photons would be increased dramatically if we were able to probe such low values of $\chi$ with the next generation of laser LSW experiments.

Fortunately, this seems doable. The current state-of-the-art LSW experiment ALPS, exploiting an optical resonator at the generation side of the experiment, resulting in a power of $\sim 1.2 \mathrm{~kW}$ available for $\gamma \rightarrow$ WISP conversions, established an upper limit $P_{\mathrm{LSW}} \lesssim$ few $\times 10^{-25}$ on the LSW probability, corresponding to an upper limit $\chi \lesssim$ few $\times 10^{-7}$ in the meV mass range. Exploiting additionally a high finesse $\left(\sim 10^{4}\right)$ optical resonator also on the regeneration side of the experiment $[30,31]$ and a single-

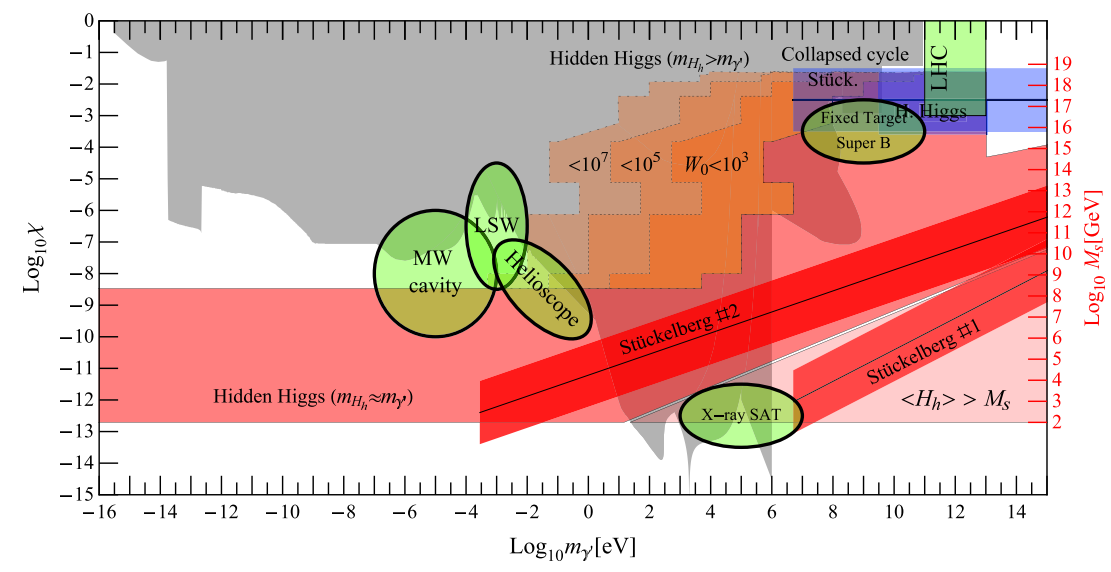

FIGURE 4. Prediction of hidden photon kinetic mixing $\chi$ with the visible photon vs. its mass $m_{\gamma^{\prime}}$ from LARGE volume string compactifications. The grey area is excluded by hidden photon searches alone. The bright red region predicted for hyperweak hidden photons whose mass arises from a hidden Higgs mechanism takes already into account the astro and cosmo constraints from minicharged particles [28]. (Compilation from Ref. [25].). 
photon counter, together with an increased power buildup, by a factor of $\sim 100$, on the generation side, it seems possible to improve the sensitivity on the LSW probability by $\sim 4+2+2=8$ orders of magnitude, corresponding to an improved sensitivity in $\chi$ by $\sim 8 / 4=2$ orders of magnitude, down to the most interesting values, $\chi \sim$ few $\times 10^{-9}$. Such values, at somewhat smaller masses, can also be probed by microwave cavity variants of the LSW technique [32, 33, 34], which are currently set up [35, 36, 37], and, at somewhat larger masses, by especially designed helioscopes to search for solar hidden photons [38], which are also under consideration (see, e.g., Ref. [39]).

Let us turn now to axions and ALPs. Although much less model dependent [40], the values of the two photon coupling $g$ of ALPs probed by the current generation of LSW experiments, $g \gtrsim$ few $\times 10^{-8} \mathrm{GeV}^{-1}$, for masses below an meV, falls short, by nearly
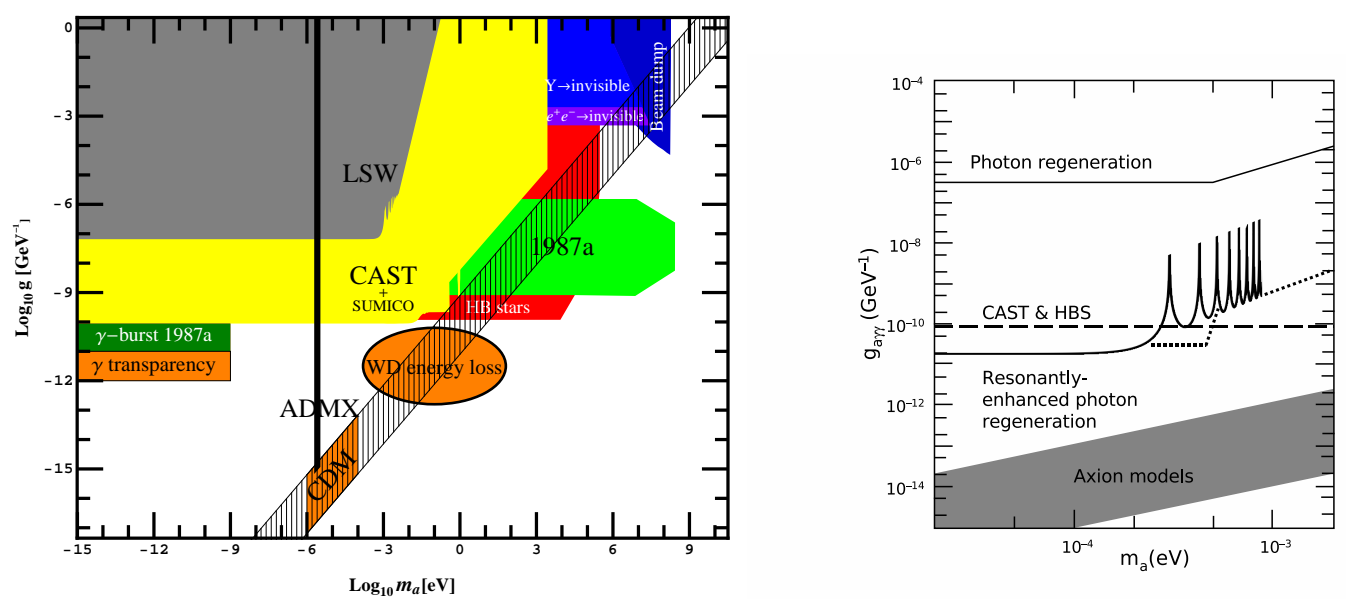

FIGURE 5. Left: Summary of cosmological and astrophysical constraints for axion-like-particles (two photon coupling $g$ vs. mass $m_{a}$ of the ALP). Note that the mass region, where the axion can be the cold dark matter (the orange regions labeled "CDM"), can be extended towards smaller masses by anthropic reasoning. Also other areas with interesting astrophysical hints, e.g. the one for a non-standard energy loss in white dwarfs [45] or the one for an anomalous $\gamma$-ray transparency of the universe [46, 47], are marked in orange. The parameter range for the axion is shown hatched. Note that the limit from the microwave cavity axion dark matter search experiment ADMX [48] is valid only under the assumption that the local density of ALPs at earth is given by the dark matter density. (Compilation from Ref. [4], where also details can be found.) Right: Prospected sensitivity of a laser LSW experiment exploiting $6+6$ Tevatron magnets and resonantly enhanced photon regeneration [49].

three orders of magnitude, to the strong limits established by lifetime considerations of horizontal branch stars $[41,42]$ and by limits on photon regeneration due to solar ALPs reported by the helioscopes CAST [43] and SUMICO [44] (cf. Fig. 5 (left)). Here, the next generation of LSW experiments has to gain about three orders of magnitude in the coupling to start to enter in previously unexplored territory. In addition to above mentioned improvements from the laser and detector side, one has to increase $B \times L$, the magnitude times the length of the magnetic field region, by one order of magnitude compared to the current experiments, e.g. by exploiting 5+5 HERA magnets at ALPS, instead of the current $1 / 2+1 / 2$ configuration. With such improvements, a sensitivity in the $g \sim$ few $\times 10^{-11} \mathrm{GeV}^{-1}$ range, for light ALPs, $m_{\phi} \ll \mathrm{meV}$, should be achievable [49, $50,51,52$ ]. For the sensitivity of a similar setup proposed in Ref. [49], exploiting 6+6 Tevatron magnets, see Fig. 5 (right). 
An even wider range of opportunities for discovery would open up if the sensitivity in $g$ can be improved even more, by one order of magnitude, down to $g \sim$ few $\times$ $10^{-12} \mathrm{GeV}^{-1}$, possibly by a combination of laser and magnet upgrades.

First of all, ALPs with such a coupling may be motivated from a top-down perspective arising from string theory. In fact, massless ALPs, with coupling to photons in the $g$ $\alpha / M_{S} \sim 10^{-12} \div 10^{-11} \mathrm{GeV}^{-1}$ range, could occur naturally in string compactifications with an intermediate string scale $M_{s} \sim 10^{9} \div 10^{10} \mathrm{GeV}$.

Secondly, there are a number of puzzling astronomical observations which may be commonly explained by cosmic photon oscillations into very light ALPs with $g$ in the above range (cf. also Fig. 5 (left)). Indeed, photons emitted by distant sources and propagating through cosmic magnetic fields can oscillate into ALPs, with a number of consequences in different situations (see, e.g., Refs. [53, 54, 55, 56, 57, 58, 59, 60, 61, 62, 63]). Interestingly, ALPs may leave their imprints in luminosity relations of active galactic nuclei $[64,65]$. In fact, mixing between photons and ALPs in the random magnetic fields in galaxy clusters induces a characteristic scatter in the relations of X-ray vs. optical luminosities of compact sources in these clusters. Evidence for such an effect has recently been found in an analysis of luminosity relations of about two hundred active galactic nuclei, providing a strong hint for the possible existence of a very light, $m_{\phi} \lesssim 10^{-12} \mathrm{eV}$, ALP, with a coupling in the $g \sim 10^{-12} \div 10^{-11} \mathrm{GeV}^{-1}$ range.

This range is also the sensitivity of another astrophysical probe of ALPs, namely the spectra of cosmologically distant $\mathrm{TeV} \gamma$-ray sources. In fact, recent observations of a few of them by ground-based gamma ray telescopes have revealed a surprising degree of transparency of the universe to very high-energy photons, which seems to point to less absorption due to pair production, may be due to a less dense extragalactic background light and/or to a harder injection spectrum at the sources than initially thought. However, there is also the intriguing possibility to explain this puzzle through photon $\leftrightarrow$ ALPs oscillations in the cosmic magnetic fields, again requiring a coupling in the $g \sim 10^{-12} \div 10^{-11} \mathrm{GeV}^{-1}$ range [46, 47] (cf. Fig. 5 (left)). The present status of this affair is far from conclusive, however. It seems that much more data from many more quite distant $\mathrm{TeV}$ gamma sources along different directions in the sky has to be collected before one may be able to perform a systematic search for hints of ALPs [66]. For this increase in statistics, we have to wait, however, for the realization of the big $\mathrm{TeV}$ gamma ray array CTA. It would be great, if we were able to probe the same range of parameters even earlier in the laboratory, by laser light shining through a wall!

\section{REFERENCES}

1. S. Weinberg, Phys. Rev. Lett. 40, 223 (1978); F. Wilczek, Phys. Rev. Lett. 40, 279 (1978).

2. L. B. Okun, Sov. Phys. JETP 56, 502 (1982) [Zh. Eksp. Teor. Fiz. 83, 892 (1982)].

3. B. Holdom, Phys. Lett. B 166, 196 (1986).

4. J. Jaeckel and A. Ringwald, arXiv:1002.0329 [hep-ph].

5. J. Jaeckel, these proceedings

6. E. Zavattini et al. [PVLAS Collaboration], Phys. Rev. Lett. 96, 110406 (2006).

7. L. Maiani, R. Petronzio and E. Zavattini, Phys. Lett. B 175, 359 (1986).

8. H. Gies, J. Jaeckel and A. Ringwald, Phys. Rev. Lett. 97, 140402 (2006).

9. M. Ahlers, H. Gies, J. Jaeckel and A. Ringwald, Phys. Rev. D 75, 035011 (2007).

10. M. Ahlers, H. Gies, J. Jaeckel, J. Redondo and A. Ringwald, Phys. Rev. D 76, 115005 (2007). 
11. M. Ahlers, H. Gies, J. Jaeckel, J. Redondo and A. Ringwald, Phys. Rev. D 77, 095001 (2008).

12. P. Sikivie, Phys. Rev. Lett. 51, 1415 (1983) [Erratum-ibid. 52, 695 (1984)].

13. A. A. Anselm, Phys. Rev. D 37, 2001 (1988).

14. K. Van Bibber et al., Phys. Rev. Lett. 59, 759 (1987).

15. C. Robilliard et al., Phys. Rev. Lett. 99, 190403 (2007).

16. A. S. Chou et al. [GammeV (T-969) Collaboration], Phys. Rev. Lett. 100, 080402 (2008).

17. P. Pugnat et al. [OSQAR Collaboration], Phys. Rev. D 78, 092003 (2008).

18. A. Afanasev et al., Phys. Rev. Lett. 101, 120401 (2008).

19. M. Fouche et al., Phys. Rev. D 78, 032013 (2008).

20. A. Afanasev et al., Phys. Lett. B 679, 317 (2009).

21. K. Ehret et al. [ALPS collaboration], Nucl. Instrum. Meth. A 612, 83 (2009).

22. R. Cameron et al., Phys. Rev. D 47, 3707 (1993).

23. A. Lindner, J. Redondo, for the [ALPS Collaboration], priv. comm. and publ. (DESY 10-030) in prep.

24. E. Zavattini et al. [PVLAS Collaboration], Phys. Rev. D 77, 032006 (2008).

25. M. Goodsell, J. Jaeckel, J. Redondo and A. Ringwald, JHEP 0911, 027 (2009).

26. J. Jaeckel, J. Redondo and A. Ringwald, Phys. Rev. Lett. 101, 131801 (2008).

27. J. Redondo, JCAP 0807, 008 (2008).

28. S. Davidson, S. Hannestad and G. Raffelt, JHEP 0005, 003 (2000).

29. C. P. Burgess et al., JHEP 0807, 073 (2008).

30. F. Hoogeveen and T. Ziegenhagen, Nucl. Phys. B 358, 3 (1991).

31. P. Sikivie, D. B. Tanner and K. van Bibber, Phys. Rev. Lett. 98, 172002 (2007).

32. F. Hoogeveen, Phys. Lett. B 288, 195 (1992).

33. J. Jaeckel and A. Ringwald, Phys. Lett. B 659, 509 (2008).

34. F. Caspers, J. Jaeckel and A. Ringwald, JINST 4, P11013 (2009).

35. R. Povey, J. Hartnett and M. Tobar, arXiv:1003.0964 [hep-ex].

36. O. K. Baker, these proceedings

37. G. Rybka, these proceedings

38. S. N. Gninenko and J. Redondo, Phys. Lett. B 664, 180 (2008).

39. M. Minowa, these proceedings

40. J. Jaeckel, E. Masso, J. Redondo, A. Ringwald and F. Takahashi, Phys. Rev. D 75, 013004 (2007).

41. G. G. Raffelt, Phys. Rev. D 33, 897 (1986).

42. G. G. Raffelt and D. S. P. Dearborn, Phys. Rev. D 36, 2211 (1987).

43. S. Andriamonje et al. [CAST Collaboration], JCAP 0704, 010 (2007).

44. Y. Inoue et al., Phys. Lett. B 668, 93 (2008).

45. J. Isern, E. Garcia-Berro, S. Torres and S. Catalan, arXiv:0806.2807 [astro-ph].

46. A. De Angelis, O. Mansutti and M. Roncadelli, Phys. Rev. D 76, 121301 (2007).

47. A. De Angelis, O. Mansutti, M. Persic and M. Roncadelli, arXiv:0807.4246 [astro-ph].

48. L. D. Duffy et al., Phys. Rev. D 74, 012006 (2006).

49. G. Mueller, P. Sikivie, D. B. Tanner and K. van Bibber, Phys. Rev. D 80, 072004 (2009).

50. A. Lindner, these proceedings

51. T. Meier, these proceedings

52. G. Mueller, these proceedings

53. C. Csaki, N. Kaloper and J. Terning, Phys. Rev. Lett. 88, 161302 (2002).

54. C. Csaki, N. Kaloper, M. Peloso and J. Terning, JCAP 0305, 005 (2003).

55. A. Mirizzi, G. G. Raffelt and P. D. Serpico, Phys. Rev. D 76, 023001 (2007).

56. D. Hooper and P. D. Serpico, Phys. Rev. Lett. 99, 231102 (2007).

57. K. A. Hochmuth and G. Sigl, Phys. Rev. D 76, 123011 (2007).

58. A. Payez, J. R. Cudell and D. Hutsemekers, AIP Conf. Proc. 1038, 211 (2008).

59. M. Fairbairn, T. Rashba and S. V. Troitsky, arXiv:0901.4085 [astro-ph.HE].

60. A. Mirizzi, J. Redondo and G. Sigl, JCAP 0908, 001 (2009).

61. A. Chou, these proceedings

62. A. Payez, these proceedings

63. J. Redondo, these proceedings

64. C. Burrage, A. C. Davis and D. J. Shaw, Phys. Rev. Lett. 102, 201101 (2009).

65. C. Burrage, these proceedings

66. A. Mirizzi and D. Montanino, JCAP 0912, 004 (2009). 